ARTICLE

Received 3 Aug 2015 | Accepted 18 Dec 2015 | Published 22 Jan 2016

DOI: $10.1038 /$ ncomms10488

OPEN

\title{
The lack of the Celf2a splicing factor converts a Duchenne genotype into a Becker phenotype
}

\author{
J. Martone ${ }^{1, \star}$, F. Briganti ${ }^{1, \star}$, I. Legnini ${ }^{1}$, M. Morlando ${ }^{1}$, E. Picillo ${ }^{2}$, O. Sthandier ${ }^{1}$, L. Politano ${ }^{2}$ \& I. Bozzoni ${ }^{1,3,4}$
}

Substitutions, deletions and duplications in the dystrophin gene lead to either the severe Duchenne muscular dystrophy (DMD) or mild Becker muscular dystrophy depending on whether out-of-frame or in-frame transcripts are produced. We identified a DMD case (GS $\Delta 44$ ) where the correlation between genotype and phenotype is not respected, even if carrying a typical Duchenne mutation (exon 44 deletion) a Becker-like phenotype was observed. Here we report that in this patient, partial restoration of an in-frame transcript occurs by natural skipping of exon 45 and that this is due to the lack of Celf2a, a splicing factor that interacts with exon 45 in the dystrophin pre-mRNA. Several experiments are presented that demonstrate the central role of Celf2a in controlling exon 45 splicing; our data point to this factor as a potential target for the improvement of those DMD therapeutic treatments, which requires exon 45 skipping.

\footnotetext{
${ }^{1}$ Department of Biology and Biotechnology Charles Darwin, Sapienza University of Rome, P.le A. Moro 5, Rome 00185, Italy. ${ }^{2}$ Department of Experimental Medicine, Cardiomyology and Medical Genetics, Second University of Napoli, I Policlinico, Piazza Miraglia, Napoli 80138, Italy. ${ }^{3}$ Center for Life Nano Science@Sapienza, Istituto Italiano di Tecnologia, Viale Regina Elena 291, Rome 00161, Italy. ${ }^{4}$ Institute Pasteur Fondazione Cenci-Bolognetti, Sapienza University of Rome, P.le A. Moro 5, Rome 00185, Italy. * These authors contributed equally to this work. Correspondence and requests for materials should be addressed to I.B. (email: irene.bozzoni@uniroma1.it).
} 
$\mathrm{D}$ uchenne muscular dystrophy (DMD) is a rare neuromuscular disease characterized by substitutions, deletions and duplications in the dystrophin gene leading to frameshift and premature translation termination ${ }^{1}$. Dystrophin is a cytoskeleton protein which stabilizes the dystrophinassociated protein complex, required for muscle fibre integrity ${ }^{2}$, and controls gene expression through nNOS activation and nitric oxide-mediated HDAC2 inactivation ${ }^{3-5}$. At variance with $\mathrm{DMD}$, the milder form of the disease, the Becker muscular dystrophy (BMD), is caused by mutations, including long deletions, which do not perturb the open reading frame (ORF) integrity and produce shorter and partially functional dystrophin isoforms. Deletions in the dystrophin gene are found in $\sim 68 \%$ of DMD patients, with the region including exons 44-55 being the most commonly mutated, together with the one encompassing exons $2-20$ (ref. 6). However, the severity of the pathology is not always predictable by exon deletion, suggesting that each patient has specific deletion breakpoints that may differentially affect splicing. Moreover the 'reading frame model', in which BMD patients produce a semi-functional, internally deleted dystrophin protein, whereas DMD patients have no detectable protein, is not always respected ${ }^{7,8}$. Uncertainty in predicting the Duchenne/ Becker pathology indicates that the clinical picture should always be accompanied by a detailed molecular analysis, including transcript structure and dystrophin-associated proteins. Several data indicated that altered splicing patterns in a collection of BMD patients do not directly result from the gene defect but probably derive from modifications in trans- rather than cis-acting factors ${ }^{9}$.

The fact that BMD patients carrying extended deletions display only mild phenotypes, suggested that manipulating the splicing of the dystrophin pre-mRNA, to induce the skipping of specific exons and restore a correct reading frame, could be a powerful strategy for DMD treatment ${ }^{10-13}$. This approach has recently entered into clinical trials through the use of antisense oligonucleotides preventing exon recognition by the splicing machinery ${ }^{14,15}$.

In this work we have studied a DMD case (GS $\Delta 44$ ), in which the correlation between genotype and phenotype is not respected: even if the genetic analysis of the dystrophin gene locus showed a deletion of exon 44 , which should normally lead to DMD, the patient is now 18 years old and can still walk independently. Molecular analyses on both GS $\Delta 44$ biopsy and primary myoblasts showed the presence of tiny but consistent dystrophin levels due to endogenous skipping of exon 45 . We describe here that this phenotype is due to the lack of the Celf2a protein isoform, that we found to enhance exon 45 inclusion.

\section{Results}

A DMD patient produces dystrophin by natural exon skipping. An individual (GS $\Delta 44)$ with a DMD genotype (deletion of exon 44 which leads to an out-of-frame transcript) was under study since, quite surprisingly, his clinical and behavioural tests were in agreement more with a Becker phenotype, even if of a severe type. Supplementary Table 1 summarizes the timed tests from the age of 5 to 18: while DMD patients normally lose ambulance around the age of 12 and need ventilatory support at their late teens, GS $\Delta 44$ is 18 years old and, even if he has lost the ability to stand up from the ground, he can walk and breath normally.

A muscle biopsy, performed at the age of 15 , indicated the unexpected presence of dystrophin at low but well detectable levels (Fig. 1a). As expected for a dystrophic sample 16 regeneration was occurring: the early myogenic factor myogenin (MYOG) was still expressed, while the terminal differentiation markers, myosin heavy chain (MHC) and muscle creatin kinase $(\mathrm{MCK})^{17}$, were lower with respect to the biopsy from a healthy control (Fig. 1b,c). Regeneration was also indicated by sustained expression of the proliferative miR-31 and by reduced levels of miR-1 and miR-133, markers of mature muscle fibres ${ }^{18}$ (Fig. 1c). Since muscle biopsies can vary considerably on the basis of the inflammatory and fibrotic state of the tissue, we derived a culture of primary myoblasts and analysed dystrophin expression upon differentiation in low serum, in parallel to control myocytes derived from a healthy individual. In agreement with the biopsy sample, in vitro differentiation confirmed the ability of GS $\Delta 44$ myocytes to produce dystrophin (Fig. 1d). A scaled dilution of proteins from controls indicated that these levels corresponded to $\sim 7 \%$ of those found in healthy myocytes. Interestingly, such low levels of dystrophin paralleled a slower timing of differentiation ${ }^{16,19}$ : in fact, myogenin expression was still detectable at day 10, while MHC was reduced in comparison with control (Fig. 1d). This is in line with the notion that dystrophic cultures show a slower progression into the differentiation process ${ }^{16}$, with BMD having on average a less severe differentiation phenotype than $\mathrm{DMD}^{20,21}$.

A detailed mapping of the GS $\Delta 44$ deletion revealed a $4065 \mathrm{bp}$ deletion, encompassing exon 44, with the fusion between nucleotide 69586 of intron 43 and nucleotide 3038 of intron 44 (Supplementary Fig. $1 \mathrm{a}-\mathrm{c}$ and Supplementary Methods). Therefore, we confirmed the DMD genotype of GS $\Delta 44$.

Reverse transcription PCR (RT-PCR) analysis on RNA from the GS $\Delta 44$ biopsy and from myocytes derived from primary myoblasts indicated that in addition to the lack of exon 44, a small fraction of transcripts also skipped exon 45 (Fig. 1e). Sequencing of this product confirmed the skipping of exon 45 and the proper fusion between exon 43 and exon 46 (not shown), giving rise to an in-frame transcript coding for a shorter but functional protein ${ }^{21,22}$. Notably, the skipping of exon 45 is currently conceived as a possible approach for the therapeutic treatment of $\Delta 44$ deletions and for those DMD mutations that through exon 45 skipping could recover the correct reading frame $^{22}$. The presence of sub-optimal splice junctions in exon 45 of the DMD gene of GS $\triangle 44$ was excluded by PCR amplification and sequence analysis of the genomic DNA (data not shown).

Because of the limited ability of in vitro propagation of primary myoblasts, fibroblasts derived from the GS $\Delta 44$ biopsy and trans-differentiated with $\mathrm{MyoD}$ towards the myogenic lineage ${ }^{21}$ were also used. These cells produced exon 45 skipping and dystrophin synthesis similarly to differentiated primary myoblasts, even if with a delayed time of expression (Fig. 2a and Supplementary Fig. 2a). Notably, two different transdifferentiated $\triangle 44$ DMD myocyte cultures, carrying unrelated deletions around exon 44 ( $\Delta 44-1$ and $\Delta 44-2)$, did not show any detectable exon 45 skipping (Fig. 2a).

GS $\Delta 44$ myoblasts lack the Celf2a splicing factor. RNA-seq experiments, performed on healthy control, GS $\Delta 44$ and $\Delta 44-1$ myoblasts and myocytes (deposited at Gene Expression Omnibus, GSE70389), were queried for the abundance of splicing enhancers (SRSF1, SRSF2, SRSF5 and SRSF6) whose potential binding motifs were identified on exon 45 by using the ESE Finder tool (ref. 23 and Supplementary Fig. 2b). This analysis indicated that abundance of such mRNAs did not vary among the different samples; finding that was validated by RT-PCR (Supplementary Fig. 2c). Therefore, none of these factors could be considered a good candidate for the expected phenotype.

Other possible candidates, among the factors previously known to change during muscle differentiation, were searched. Among them, the CUGBP, Elav-like family member 2 (Celf2) protein was demonstrated to regulate various processes including alternative splicing, editing, RNA stability and translation ${ }^{24-29}$ and suggested to participate in dystrophin splicing ${ }^{9}$. CELF2 is composed of 
a

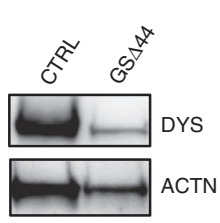

b

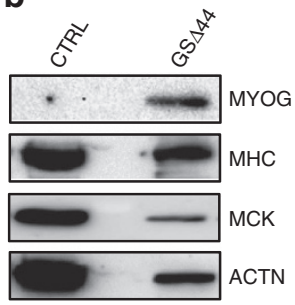

C

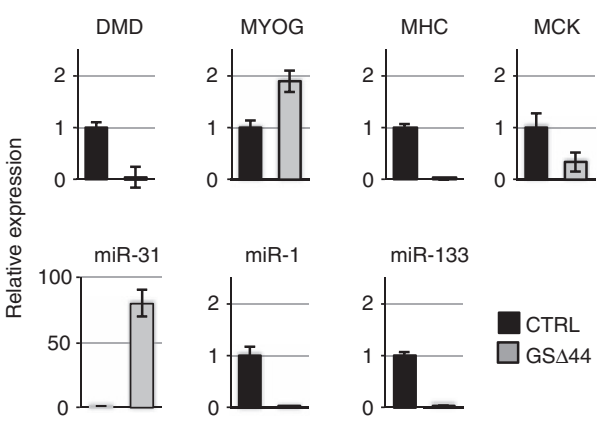

d

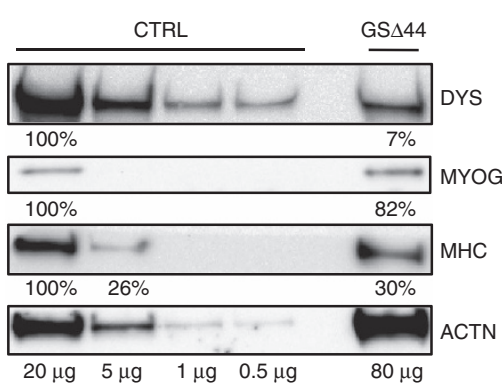

e

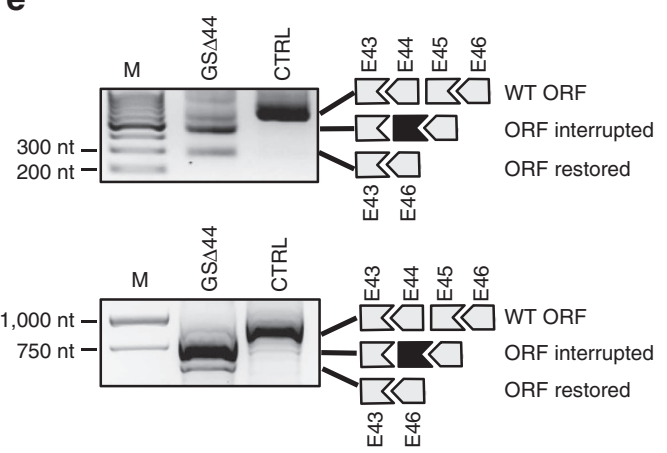

Figure 1 | Expression of dystrophin and myogenic markers in $\mathbf{G S} \mathbf{\Delta} \mathbf{4 4}$ biopsy and primary myoblasts. (a,b) Western blot on proteins (20 $\mu \mathrm{g})$ extracted from human muscle biopsies of a healthy control (CTRL) and from GS 444 probed with antibodies against dystrophin (DYS, a), or myogenin (MYOG), MHC and muscle creatine-kinase (MCK, b). Actinin (ACTN) was used as a loading control. (c) Quantitative RT-PCR of myogenic markers and myomiRs performed on RNA extracted from healthy control (CTRL) and GS $\triangle 44$ muscle biopsies. Bar plots show relative expression levels with respect to control set to 1: values were normalized with GAPDH and hypoxanthine phosphoribosyltransferase (HPRT) for mRNAs and with U6 and miR-16 for miRNAs. Bars indicate a confidence level of 95\%. (d) Western blot on proteins extracted from healthy control (CTRL) and GS $\Delta 44$ primary myoblasts upon 10 days of shift to differentiation conditions and probed with antibodies against dystrophin (DYS), myogenin (MYOG) and MHC. The amount of proteins loaded is indicated below each lane. Actinin (ACTN) was used as a loading control. (e) Nested RT-PCR performed on RNA from biopsy samples (upper panel) and differentiated myocytes obtained as in $\mathbf{d}$ (lower panel); $20 \mathrm{ng}$ of cDNA were amplified with primers located in exons 42 and 53 and then with primers in exons 43 and 46 (upper panel) and in exons 43 and 48 (lower panel). A schematic representation of the WT, interrupted and restored ORFs is shown on the side. $\mathrm{M}$, molecular weight marker: GeneRuler $100 \mathrm{bp}$ (Thermo Scientific) for the upper panel and GeneRuler $1 \mathrm{~kb}$ (Thermo Scientific) for the lower panel; sizes are shown on the side.

three isoforms differing by their first exon (exons $1 \mathrm{a}, 1 \mathrm{~b}$ and $1 \mathrm{c})^{9}$, that are here termed CELF2a, b and c, respectively (Supplementary Fig. 2d); moreover, CELF2a has a variant isoform consisting in the alternative use of a $18 \mathrm{nt}$ extended exon 11 (ref. 28). Analysis of CELF2 transcripts in the RNA-seq of control, GS $\Delta 44$ and $\Delta 44-1$ samples indicated that while the CELF2b and CELF2c isoforms were expressed in all samples, CELF2a was completely absent in GS $\Delta 44$ both in growth (GM) and differentiation (DM) conditions.

The absence of CELF2a mRNA, was validated by RT-PCR both in the biopsy sample (Fig. 2b) and in myoblasts (GM) and myocytes (DM) of GS $\Delta 44$ (Fig. 2c). Notably, all three isoforms were instead present in control and $\Delta 44-1$ cells (Fig. 2c). Western blot analysis with commercially available antibodies indicated their inability to distinguish between the three different isoforms (Supplementary Fig. 2e); therefore, in following analyses CELF2 isoform expression was always tested at the RNA level.

When analysing trans-differentiated fibroblasts of GS $\Delta 44$ 's parents, it was interesting to find that while CELF2a is present in the father, the mother specifically lacks this isoform (Fig. 2d) and displays skipping of exon 45 (Supplementary Fig. 2f). In her case, the skipped product is present at very low levels since exclusion of exon 45 would produce an out-of-frame transcript that undergoes degradation through the NMD pathway.

A $1.3 \mathrm{~kb}$ DNA sequence upstream the TSS and $200 \mathrm{nt}$ of the first exon/intron of CELF2a was sequenced in the GS $\triangle 44$ DNA.
We identified only two variants of annotated single-nucleotide polymorphisms (chr10:11046386 T/C, chr10:11047206 C/G of the hg19 human assembly), one of which was laying at the border of a CpG island; neither sequence corresponded to obvious transcriptional factor binding sites as checked on the Encode Transcriptional factors binding tracks at UCSC genome browser and on TransFac database. To check whether methylation could affect CELF2a expression, GS $\Delta 44$ myoblasts were treated for 7 days with 5-azacytidine in parallel with control myoblasts. No rescue of CELF2a expression was obtained in treated versus untreated GS $\Delta 44$ cells, suggesting that likely promoter methylation is not the cause of CELF2a lack in these cells (Supplementary Fig. 2g). Also in control cells, 5-azacytidine treatment did not affect CELF2a levels (Supplementary Fig. $2 \mathrm{~g}$ and Supplementary Methods).

To detect similar exon skipping events possibly related to the absence of CELF2a, the RNAseq data obtained from control, $\Delta 44$ and GS $\Delta 44$ myoblasts and myocytes were analysed by MATS (ref. 30). Ratios of exon inclusion/skipping were compared in GS $\Delta 44$ versus $\Delta 44-1$ and in GS $\Delta 44$ versus control (Supplementary Table 2 and Supplementary Data 1). To exclude exon skipping events related to the pathological state or to altered differentiation of DMD cells, we selected only those that were concordant and significant in both GS $\Delta 44$ versus control and GS $\Delta 44$ versus $\Delta 44-1$. Out of 29 genes, correlating with the lack of CELF2a (Supplementary Table 2), five transcripts exhibited 
a

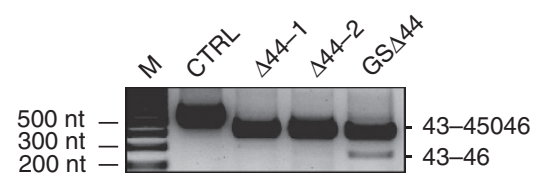

C
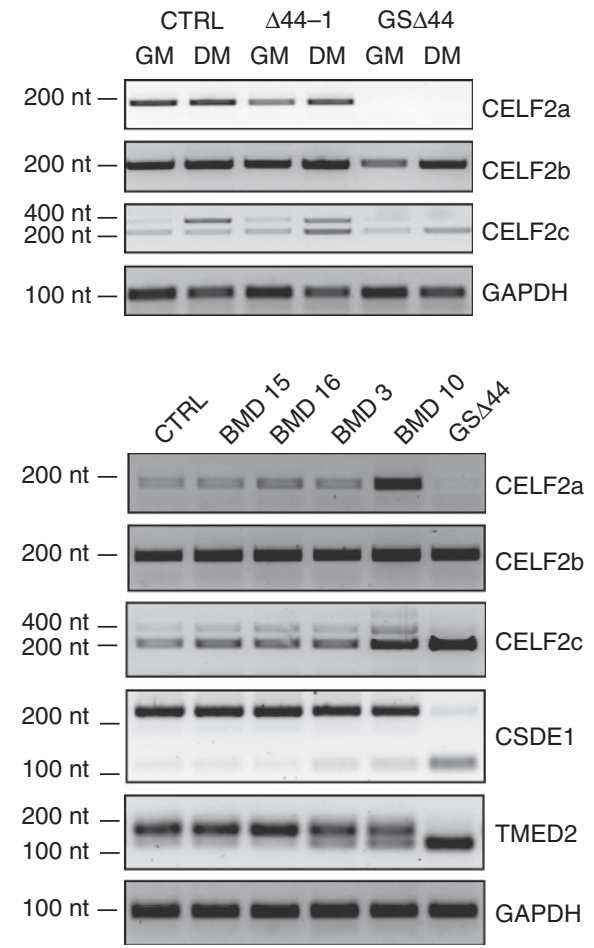

b

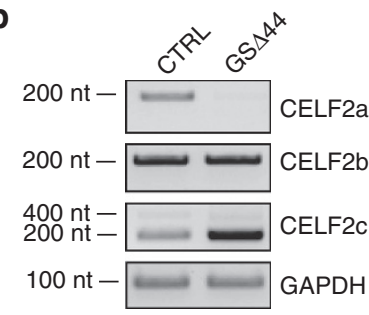

d

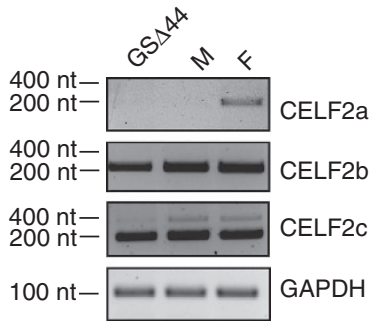

f

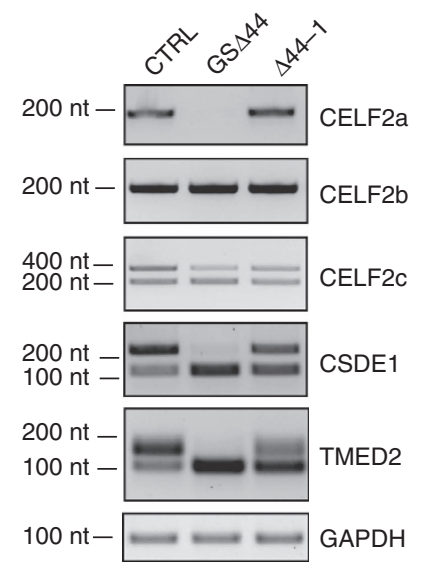

Figure 2 | GS $\Delta 44$ lacks the CELF2a isoform. (a) Fibroblasts from control (CTRL), two $\Delta 44$ unrelated DMD ( $\Delta 44-1$ and $\Delta 44-2)$ and GS $\Delta 44$ biopsies were trans-differentiated into myocytes via infection with a lentivirus containing a MyoD-expression cassette. After 10 days in differentiation medium, RNA was extracted and RT-PCR was performed with primers located in exons 43 and 46. M, molecular weight marker: GeneRuler 100 bp (Thermo Scientific). (b) RT-PCR for the three CELF2 isoforms on RNA from control (CTRL) and GS 444 biopsies. (c) MyoD-transduced fibroblasts from control (CTRL), $\triangle 44-1$ and GS $\triangle 44$ were maintained in growth conditions (GM) or shifted to differentiation medium (DM) for 10 days. RNA was extracted and analysed by RT-PCR with oligos specific for the three CELF2 isoforms. (d) MyoD-transduced fibroblasts from the mother (M) and father (F) of GS $\triangle 44$ were induced to differentiate and RNA analysed by RT-PCR for the three CELF2 isoforms. (e) RT-PCR for the three CELF2 isoforms and for CSDE1 and TMED2 mRNAs on RNA from control (CTRL), GS 444 and 4 different BMD biopsies (BMD-3, BMD-10, BMD-15 and BMD-16). (f) RT-PCR for the three CELF2 isoforms and for CSDE1 and TMED mRNAs on RNA from control (CTRL), $\Delta 44-1$ and GS $\triangle 44$ trans-differentiated myocytes. In all experiments GAPDH was used as control. Molecular weight marker: GeneRuler 100 bp (Thermo Scientific).

alternative splicing events in differentiated myocytes and two of them were experimentally validated, CSDE1 and TMED2. In comparison with control and $\Delta 44-1$, GS $\Delta 44$ shows the preferential accumulation of skipped isoforms for both CSDE1 and TMED2 transcripts (Supplementary Fig. 2h,i).

Four unrelated BMD biopsies ${ }^{21}$ (Supplementary Fig. 2j) were then analysed; they all expressed CELF2a and displayed the same CSDE1 and TMED2 isoforms as the control sample (Fig. 2e). The same isoforms' pattern was also obtained in $\Delta 44-1$ myocytes (Fig. 2f). In contrast, the GS $\Delta 44$ biopsy and myocytes, besides lacking CELF2a, showed only the skipped isoforms for both CSDE1 and TMED2 (Fig. 2e,f). Notably, while the isoforms present in control and BMD are found in mature muscle fibres (Supplementary Table 2), those present in GS $\Delta 44$ correspond to more ubiquitous species ${ }^{31}$. In fact, comparison of CSDE1 and TMED2 in wild-type (WT) and GS $\Delta 44$ fibroblasts and myoblasts, indicated that only the unskipped species are the myocyte-specific isoforms (Supplementary Fig. 2k). The skipped splicing variants detected in GS $\Delta 44$ do not have premature stop codons and maintain well-defined protein coding ability even if they are not specific for muscle cells. From these data it cannot be excluded whether any of these changes in isoform ratio could have secondary detrimental effects, even though GS $\Delta 44$ does not show any adverse effect in any other specific physiological activity.

Celf2a affects exon 45 splicing. We next tested whether rescue of Celf2a in GS $\Delta 44$ could affect exon 45 splicing. A Flag-CELF2a construct (pFlag-CELF2a-1) or an empty vector (pcDNA) were transfected into GS $\Delta 44$ myoblasts and exon 45 skipping was analysed upon induction of differentiation. Efficient expression of CELF2a was confirmed in GS $\Delta 44$ myocytes both at the RNA and protein levels (Fig. 3a). Notably, under these conditions, exon 45 skipping was prevented (Fig. 3a). The same result was obtained when using a plasmid encoding for the CELF2a variant, lacking the $18 \mathrm{nt}$ extension in exon 11 (pCELF2a-2, Supplementary 
a
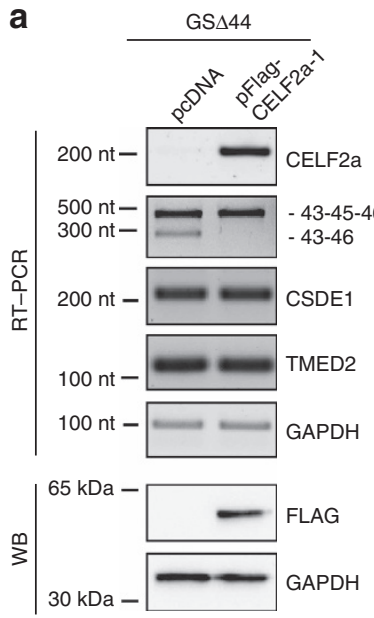

e

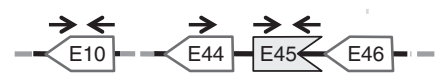

DMD pre-mRNA

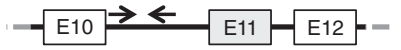

IR pre-mRNA b

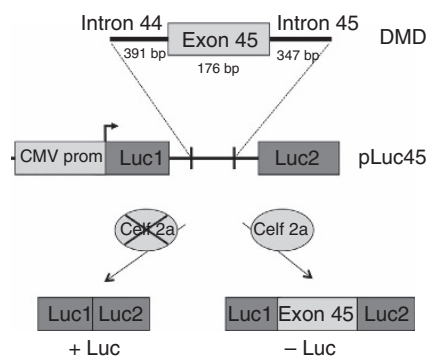

c

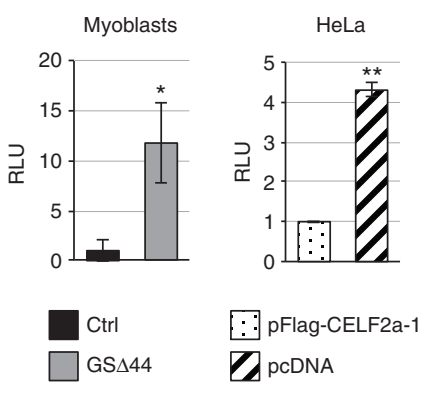

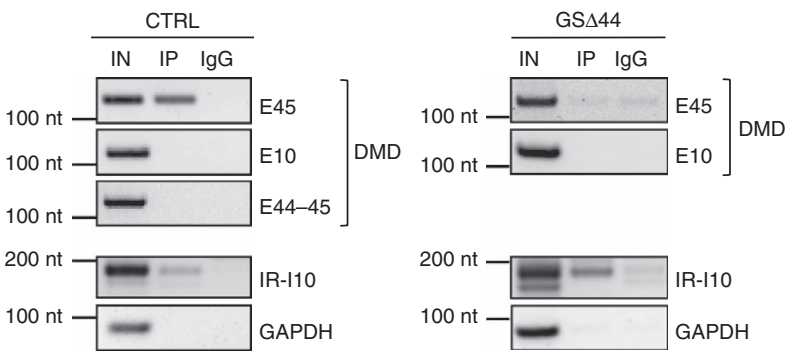

Figure 3 | CELF2a favours exon 45 inclusion. (a) GS $\triangle 44$ trans-differentiated myocytes were transfected with an empty vector (pcDNA) or with a Flag-CELF2a construct (pFlag-CELF2a-1). Cells were shifted to DM medium for 10 days and the RNA analysed by RT-PCR for Flag-CELF2a expression, for exon 45 skipping (43-46) and for CSDE1 and TMED2 isoforms (upper panel). The expression of the exogenous Flag-CELF2a was tested by western blot with anti-FLAG antibodies (lower panel). GAPDH was used as loading control. (b) Schematic representation of the pLuc 45 construct: the DMD gene region encompassing exon 45 plus portions of the flanking introns was cloned into the intron of pcDNA3.1-Luc. This plasmid contains the CMV promoter driving the expression of a Firefly luciferase pre-mRNA that produces luciferase only upon splicing (lower part). (c) The Bar plots show the relative luminescence units (RLU) of Firefly luciferase in control (black bar) and GS 444 (grey bar) myoblasts transfected with pLuc 45 and incubated $48 \mathrm{~h}$. The values were normalized for Renilla luciferase activity derived from a co-transfected pRL-TK plasmid. Error bars represent standard error from two independent experiments. ${ }^{*} P<0.05$. (d) The Bar plots show the RLU of Firefly luciferase in HeLa cells transfected with pLuc45 and either pFlag-CELF2a-1 (dotted bar) or pcDNA (dashed bar) and incubated $48 \mathrm{~h}$. The values were normalized for Renilla luciferase activity derived from a co-transfected pRL-TK plasmid. Error bars represent s.e.m. from three independent experiments. ${ }^{\star \star} P<0.01$. (e) Left panel: schematic representation of part of the dystrophin (DMD) and insulin receptor (IR) pre-mRNAs. Arrows indicate the positions of the oligos used. Right panels: control (CTRL) and GS $\triangle 44$ myocytes were UV crosslinked and subjected to nuclear-cytoplasmic fractionation. Nuclear extracts were immunoprecipitated by anti-CELF2 (lane IP) and control IgG antibodies. The presence of the regions indicated on the right (E45, E44-45 and E10 for DMD; IR-110 for IR) was analysed by RT-PCR. GAPDH was used as negative control. 'IN' samples represent $10 \%$ of the total nuclear extract. Molecular weight marker: GeneRuler 100 bp (Thermo Scientific). CMV, cytomegalovirus.

Fig. 3a). Interestingly, the overexpression of either forms of CELF2a did not produce any change in the splicing pattern of CSDE1 and TMED2 (Fig. 3a) indicating that Celf2a has no effect on these two substrates. These results open the interesting question whether CELF2a, together with CSDE1 and TMED2, are on a common pathway perhaps responding to a common upstream effector.

To further analyse Celf2a splicing activity, $\Delta 44-1$ myoblasts were infected with a lentiviral construct expressing shRNAs against CELF2a and induced to differentiate for 10 days in the presence of anti-CELF2a LNA GapmeRs. With respect to a $30 \%$ decrease of CELF2a, a little amount of exon 45 skipping was observed, indicating again the ability of this factor to impinge specifically on exon 45 splicing in a myogenic environment (Supplementary Fig. 3b and Supplementary Methods).

Celf2a activity was also tested on an artificial construct (pLuc45, Fig. 3b) containing exon 45 and portions of the flanking regions cloned inside the intron of pcDNA3.1-Luc (refs 32,33). From pLuc45, luciferase can be produced only when exon 45 is skipped. When GS $\Delta 44$ myoblasts were transfected with pLuc 45 , a 10 -fold increase in luciferase activity with respect to control cells was obtained (Fig. 3c). These data indicate that the absence of CELF2a leads to exon 45 skipping also in this artificial construct and that the regions included are sufficient to respond to Celf2a activity. The effects of CELF2a absence were also tested in a cellular context devoid of CELF2a, such as HeLa cells (Supplementary Fig. 3c). Therefore, pLuc45 was transfected into HeLa cells together with pFlag-CELF2a or with an empty vector as control ( $\mathrm{pcDNA})$. The results show that while very little luciferase activity is produced upon ectopic expression of CELF2a (Supplementary Fig. 3d), in control cells luciferase levels increase by four-fold (Fig. 3d).

Finally, to test whether Celf2a interacts with the region containing exon 45, a cross-linking immunoprecipitation (CLIP) assay was performed on nuclear RNA from control and GS $\Delta 44$ myocytes. As a positive control, the region inside intron 10 of the insulin receptor gene $(I R)$, previously reported to bind Celf2c (refs 25,34), was used. Figure $3 e$ shows that, in control myocytes (panel CTRL), Celf2 antibodies are able to immunoprecipitate the region containing exon 45 of the dystrophin pre-mRNA (E45) as well as a specific region inside intron 10 of the IR transcript (IR-I10). No interaction was instead detected 
on regions containing exon 10 (E10) of the dystrophin premRNA or when amplification of the spliced exons 44-45 (E4445) region was performed. Notably, when CLIP was performed on GS $\Delta 44$ myocytes (panel GS $\Delta 44$ ), no signal was found on the dystrophin exon 45 containing region, while interaction was detected on intron 10 of the IR transcript (Fig. 3e). Since GS $\Delta 44$ myocytes express only the CELF2b and $\mathrm{c}$ isoforms, these results indicate that exon 45 interaction in control cells is specifically due to CELF2a. In conclusion, these data indicate that the Celf2a isoform is able to interact with the dystrophin pre-mRNA at the level of exon 45 and to enhance its inclusion during the splicing reaction.

\section{Discussion}

The exon skipping strategy, as a therapeutic approach for DMD, relies on the concept of converting severe Duchenne phenotypes into milder Becker ones. In line with this, several studies have shown that the disease course of BMD patients with a mutation mimicking a 'skipped' DMD mutation is relatively mild even though BMD individuals display a large range of phenotypes ${ }^{35}$, likely reflecting the ability of shorter dystrophin proteins to accomplish only a subset of the different functions of the WT full-length form ${ }^{8}$ and to be able to produce different amounts of dystrophin. This strong variability stands for individual heterogeneity likely including the specific deletion as well as possible trans-acting factors 9 .

Moreover, one of the major concerns about the exon skipping approach resides on how much dystrophin should be required in order to be of therapeutic benefit. Here we show a DMD patient case of a 18 -year-old boy, with a $\Delta 44$ mutation; however, his clinical features, are in net contrast with the common lost of deambulation ability of DMD patients occurring around the first decade of age. Surprisingly, GS $\Delta 44$ myocytes, through spontaneous exon skipping, produce $7 \%$ of dystrophin with respect to control cells, indicating that this amount of dystrophin is enough to alleviate the severity of the disease. Therefore, GS $\Delta 44$ is an exemplary human case indicating that even very low levels of dystrophin might provide milder dystrophic conditions. GS $\Delta 44$ presents an intermediate phenotype between a typical DMD patient and a Becker individual, who can be asymptomatic for several decades; in fact, GS $\Delta 44$ walks independently even if has lost the ability to stand up from the ground. This is in line with the finding that levels of dystrophin slightly higher than those of GS $\Delta 44$ were found in a large cohort of BMD individuals ${ }^{35}$ and required in mice to slow down or prevent disease progression and improve overall muscle function ${ }^{36,37}$.

By transcriptome analysis of GS $\Delta 44$ myocytes, we discovered the absence of CELF2a, a specific isoform of the Celf 2 splicing factor and we were able to correlate the absence of this product with exon 45 skipping occurring in GS $\Delta 44$. CELF2 mRNA is expressed at variable levels in BMD/DMD skeletal muscles and its role in splicing regulation was previously suggested ${ }^{9}$. Celf2 belongs to the CELF/Bruno-like family of RNA-binding proteins that, in mammals, contains six members. This family of highly conserved proteins is involved in regulation of cell-specific and developmentally regulated alternative splicing ${ }^{29}$. The highest expression of CELF2 is found in heart, skeletal muscle and brain and single-nucleotide mutations in this gene have been correlated with a familial form of arrhythmogenic right ventricular ${ }^{28}$.

Since no mutations were found around the promoter and first exon/intron regions of the GS $\triangle 44$ CELF2a gene, it is likely that the genetic cause for its absence resides on upstream factors regulating its biogenesis or to a still unidentified epigenetic control. As other transcripts appear to be specifically affected in GS $\Delta 44$ myocytes, it will be interesting to analyse whether they are part of a common regulatory pathway specifically impinging on muscle homeostasis.

Our data suggest that CELF2a inhibition could represents an interesting new strategy to be considered for improving DMD treatment, at least in those cases where the skipping of exon 45 would result in the recovery of mRNA with an ORF. A relevant challenge for the future would be to design and set up genetic or pharmacological treatments for interfering with Celf2a activity; moreover, these results point to the relevance of studying the genomic milieu of different patients in order to facilitate the clinical development of personalized therapies.

\begin{abstract}
Methods
Case report. The proband is a 18-year-old boy, the first child of non-consanguineous Italian healthy parents. There was no family history of autoimmune or musculoskeletal disorders in the family. The birth was at term, by caesarean delivery. The baby presented neonatal jaundice treated by phototherapy. The child has never crawled and has acquired independent walking at 18 months. At 1 month of age he presented intolerance to breast milk so he began to take formula. At 7 months he presented an episode of bronchospasm that required hospitalization at the department of pediatrics of the local hospital. During hospitalization, laboratory tests pointed out high levels of transaminases (GOT $797 \mathrm{Ul}^{-1}$ and GPT $\left.266 \mathrm{Ul}^{-1}\right)$ and creatin kinase $\left(12622 \mathrm{Ul}^{-1}\right)$. Because this elevation, the family has been addressed at the Cardiomyology and Medical Genetics of Second University of Naples suspecting a muscle disorder, where he arrived at the age of 8 months. At the first control, the baby did not show pseudo-hypertrophy of the calves, but given the high values of the creatin kinase, a blood sample was carried out, with the mother's consent, to perform the molecular analysis of the dystrophin gene. The analysis showed the isolated deletion of the exon 44, an out-of-frame mutation consistent with a diagnosis of DMD. Since then, the child underwent regular checks every 6 months, by the same clinical team. Despite the prognosis, the evolution of the disease was very mild, at the age of 5 years the boy was still able to stand up from the floor in $5 \mathrm{~s}$, though showing the Gowers manoeuvre, and to climbing stairs without support in $2 \mathrm{~s}$. The electrocardiogram showed signs of postero-lateral fibrosis, a picture typical of dystrophinopathic cardiomyopathy ${ }^{38}$ so that the administration of steroids (Deflazacort $-0,75 \mathrm{mg} \mathrm{kg}^{-1} \mathrm{day}^{-1}$ ) and Ace-inhibitors (Fosinopril $5 \mathrm{mg}$ day $^{-1}$ ) was started, according to standard protocol.

At the last control—at the age of 18 years-the boy walks independently, with a waddling gait, but shows some difficulty in getting up from a chair and climbing stairs. North Star (NSAA) has a value of $8 / 34$ and he was able of walking $252 \mathrm{~m}$ in $6 \mathrm{~min}(6 \mathrm{MWT})$. The electrocardiogram showed a progression of the fibrosis, while echocardiography showed normal diameters and volumes of cardiac chambers, normal thickness of septum and left posterior wall and a normal ejection fraction. There was a mild reduction of the vital capacity values (FVC $2610 \mathrm{ml}, 61 \%$ ).
\end{abstract}

Skeletal muscle biopsies. BMD-3, BMD-10, BMD-15, BMD-16 and control skeletal muscle biopsies were obtained, with informed consent, from the Laboratory of Molecular Medicine, Department of Neuroscience, Bambino Gesù Children's Hospital, Rome whereas SG $\Delta 44$ biopsy was obtained from the EuroBiobank and Telethon Neuromuscular Biobank of Second University of Napoli.

Isolation of myoblasts from muscle biopsies. Muscle tissue was minced and placed at $37^{\circ} \mathrm{C}$ for $45 \mathrm{~min}$, with gently shaking, in $20 \mathrm{ml}$ of phosphate-buffered saline (PBS)-collagenase solution $\left(1 \mathrm{mg} \mathrm{ml}^{-1}\right)$. Resuspended solution was then passed throught a $70 \mu \mathrm{m}$ filter. After centrifugation (1500 r.p.m. for $10 \mathrm{~min}$ at $4{ }^{\circ} \mathrm{C}$ ) the pellet was resuspended in RPMI, $15 \% \mathrm{FBS}, 1 \mathrm{X}$ P/S, plated in collagenate plates and incubated at $37^{\circ} \mathrm{C}$ with $5 \%$ of $\mathrm{CO}_{2}$.

Cell cultures and treatments. WT (9208), $\Delta 44-1$ (9981) (from the Telethon Network of Genetic Biobanks) and GS $\Delta 44$ human primary myoblasts were cultured in Human skeletal muscle growth medium (PromoCell) and grown in a humidified incubator, at $5 \% \mathrm{CO}_{2}$ and $37^{\circ} \mathrm{C}$. Cells were induced to differentiate with human skeletal muscle differentiation medium (PromoCell). Transient transfection of plasmids was carried out with the use of Lipofectamine-2000 (Invitrogen) according to the manufacturer's specifications.

Reprogramming of fibroblasts into myoblasts. Conversion of fibroblasts into myoblasts was achieved by ectopic expression of $M y o D$ gene $^{39,40}$ through lentiviral infection.

Virus preparation and cell transduction. Viruses were prepared as described ${ }^{33}$. Cells were transduced with $5 \times 10^{6}$ lentiviral particles for $5 \mathrm{~h}$ and harvested after 10 days of differentiation. 
DNA and RNA preparation. Cells were harvested in QIAzol Lysis Reagent (Qiagen) and biopsies were homogenized with a rotor-stator homogenizer in the presence of QIAzol Lysis Reagent (Qiagen). RNA was extracted by miRNeasy (Qiagen), following manufacturer's specifications; Genomic DNA was extracted from the organic phase (interphase) of QIAzol Lysis Reagent (Qiagen) after RNA preparation. Concentrations were assessed with Nanodrop ND-1000 Spectrophotometer (CELBIO; Pero, Milan, Italy).

Real-time PCR. cDNA generation was carried out using the miScript Reverse Transcription Kit (QIAGEN). Real-time PCR detection of miRNAs and mRNAs was performed using the miScript SYBR-Green PCR Kit and QuantiTect and miScript Primer Assays (QIAGEN).

RT-PCR. Reverse transcription was performed on $300 \mathrm{ng}$ of total RNA using SuperScript VILO cDNA Synthesis Kit (Life Technologies) and PCR was performed using MyTaq HS DNA Polymerase (Bioline). Oligonucleotides are listed below.
$>$ DMD E42F GAAGACATGCCTTTGGAAATTTCT
$>$ DMD E43F CTACAACAAAGCTCAGGTCG
$>$ DMD E46R CTCTTTTCCAGGTTCAAGTGG
$>$ DMD E48R CTGAACGTCAAATGGTCCTTC
$>$ DMD E53R TTGTACTTCATCCCACTGATT
$>$ CELF2 E1aF CTATGAGAAATGAAGAGCTGC
$>$ CELF2 E1bF GATTTCCTCCCGGACATGACG
$>$ CELF2 E1cF CTCTGCTCGACAGCAGCACG
$>$ CELF2 E2R GAGGACGTTGATCTGGTAGAC
$>$ TMED2 F GACTCCAAAAATAGTGATGTTCAC
$>$ TMED2 R GCTGTCATCGCCACTGCTAG
$>$ CSDE1 F GCAATATTTGAGTATCACTGCG
$>$ CSDE1 R AACCCCAGTTTCACGCAGTGC

Western blot analysis. Protein extracts were loaded onto a NuPAGE Tris-Acetate Minigel 3-8\% $1 \mathrm{~mm}$ (Invitrogen) or onto a NuPAGE Bis-Tris minigel 4-12\% $1 \mathrm{~mm}$ (Invitrogen). Running and blotting were performed in an XCell SureLock Minicell (Invitrogen) according to the manufacturer's instructions and proteins were transferred to a nitrocellulose transfer membrane (Amersham Protran).

Membranes were blocked with $10 \%$ non-fat dry milk and incubated $\mathrm{o} / \mathrm{n}$ with primary antibodies. Protein detection was carried out with Clarity ECL Western Blotting Substrate (Bio-Rad). Primary antibodies: anti-dystrophin (NCL-DYS1 Novocastra Laboratories, 1:40); anti-myogenin (sc-12732 Santa Cruz 1:1,000); antiMHC (anti-MF-20, 1:20), anti-MCK (sc-15161 Santa Cruz, 1:500 in TBST); antiactinin (ACTN sc-15335 Santa Cruz, 1:1000); and anti-glyceraldehyde-3-phosphate dehydrogenase (GAPDH) (sc-365062 Santa Cruz, 1:10,000). Anti-CELF (ab50734 Abcam, 1:1,250). Anti-FLAG M2 (F1804 Sigma-Aldrich, 1:1,000). Secondary antibodies: ImmunoPure Goat Anti-Rabbit IgG Peroxidase Conjugated (31460 Pierce, 1:5,000); ImmunoPure Goat Anti-Mouse IgG Peroxidase Conjugated (31430 Pierce, 1:10,000); donkey anti-goat IgG-HRP (sc-2020 Santa Cruz, 1:5,000). Uncropped scans of blots in Fig. 1a,b,d are shown in Supplementary Fig. 4.

Overexpression of CELF2a. Construct for the overexpression of CELF2a was obtained by cloning CELF2a coding sequence in pCDNA3.1 $(+)$ plasmid (Invitrogen). CELF2a coding sequence was amplified by PCR from WT myoblast cDNA using the oligonucleotides: 5'AAAAAAGCTTATGCGCTGTCCCAAATCC GCT- $3^{\prime}$ and $5^{\prime}$-AAAACTCGAGTCAGTAAGGTTTGCTGTCGTTTTTG- ${ }^{\prime}$.

The italicized bases are HindIII and XhoI restriction sites. pFLAG-CELF2a was obtained by inverse PCR using the oligos: $5^{\prime}$-gtccttgtagtccatAAGCTTAAGTTT AAACGCTAGC- $3^{\prime}$ and $5^{\prime}$-gacgatgacaagATGCGCTGTCCCAAATCCGCTG-3' The lower case bases are the ones coding for the FLAG tag.

pLuc45 reporter construct and luciferase assays. The insert ' 45 ' was obtained by PCR from WT genomic DNA using the oligonucleotides: $5^{\prime}$-AAGGAAAAAA GCGGCCGCAAATACTTTGTTCATGTT- ${ }^{\prime}$ and $5^{\prime}$-CCTTAATTAAACAATCAT TTATAATTGC $-3^{\prime}$. The italicized bases are NotI and PacI restriction sites. The insert, made of the whole exon 45 flanked by $391 \mathrm{bp}$ of intron 44 and $347 \mathrm{bp}$ of intron 45, was inserted into pcDNA3.1-Luc vector. Transfection efficiency of this construct was assessed by cotransfection of pRLTK plasmid (Promega) encoding for renilla luciferase gene. RLuc and FLuc activities were measured by Dual Glo Luciferase assay (Promega).

RNA sequencing and bioinformatic analysis. Total RNA from GS $\Delta 44, \Delta 44-1$ and WT myoblasts and myotubes was isolated by Qiazol reagent and purified on miRNeasy columns according to manufacturer instructions (Qiagen). Ribosomal depletion and library preparation were carried out at Istituto di Genomica Applicata (Udine) according to the Illumina Truseq protocol. Reads $(2 \times 100$ paired end) were produced on an Illumina HiSeq 2000. After base calling (Casava) and adaptor removal (Cutadapt), reads were alligned to the human genome (hg19) using TopHat 2.0.11 and the resulting bam files used as input for MATS 3.0.8.
Ensembl GRCh37 was used as reference annotation. Exon skipping/inclusion events detected by MATS with both counting spliced reads and exon coverage were then filtered for false discovery rate $<0.1$ and concordance between the following pairwise comparisons: GS $\Delta 44$ versus WT (myoblasts, GM \#A) with GS $\Delta 44$ versus $\Delta 44-1$ (myoblasts) and GS $\Delta 44$ versus WT (myotubes, DM\#A) with GS $\Delta 44$ versus $\Delta 44-1$ (myotubes).

CLIP assay. The RNP precipitation was performed using $5 \times 10^{6}$ human WT and GS $\Delta 44$ myoblasts cultured in DM medium for 5 days, rinsed with PBS and UV crosslinked once at $400 \mathrm{~mJ} \mathrm{~cm}{ }^{-2}$ in Spectrolinker XL-1000. Cell were harvested in $400 \mu \mathrm{l}$ of PBS and $1.6 \mathrm{ml}$ of Nuclear isolation buffer $(0.32 \mathrm{M}$ Sucrose; $10 \mathrm{mM}$ Tris- $\mathrm{HCl} \mathrm{Ph} \mathrm{7.5;} 5 \mathrm{mM} \mathrm{MgCl}_{2}$; and 1\% Triton X-100) and incubated for $20 \mathrm{~min}$ on ice. Nuclei were collected by centrifugation at $2500 \mathrm{~g}$ for $15 \mathrm{~min}$ and resuspended in $1 \mathrm{ml}$ of RIP Buffer $(150 \mathrm{mM} \mathrm{KCl} ; 25 \mathrm{mM}$ Tris- $\mathrm{HCl} \mathrm{Ph} 7.4 ; 5 \mathrm{mM}$ EDTA; $0.5 \mathrm{mM}$ DTT; and $0.5 \% \mathrm{NP} 40$ ). Nuclei were mechanically sheared using a dounce homogenizer and debris were eliminated by centrifugation. A total of $5 \mu \mathrm{g}$ of Anti-CELF (ab50734 Abcam) and $5 \mu \mathrm{g}$ of the control IgG antibodies were used for the immunoprecipitation for $12 \mathrm{~h}$ at $4^{\circ} \mathrm{C}$. A total of $40 \mu \mathrm{l}$ of protein $\mathrm{G}$ agarose beads were added and incubated for $2 \mathrm{~h}$ at $4{ }^{\circ} \mathrm{C}$. Beads were washed three times with RIP buffer and once with PBS. The beads were then treated with proteinase $\mathrm{K}$ (Ambion) before the addition of Tryzol for the RNA extraction. The oligonucleotides used in the RT-PCR analyses are listed below.

$>$ DMD E10F GCAGTTCATTGATGGAGAGTG

$>$ DMD E10 R CACCACTTCCACATCATTAGA

$>$ DMD E44F GATTGGACAGATCTGTTGAGA

$>$ DMD E45F ATGGCATTGGGCAGCGGCAAAC

$>$ DMD E45R ACCTCCTGCCACCGCAGATTC

$>$ IR F GCCATGTTGGCTAGGCTGGTCTC

$>$ IR R GGCTTACACCACTGCGCTCAGC

$>$ GAPDH F GGAAGGTGAAGGTCGGAGTC

$>$ GAPDH R TTACCACAGTTAAAAGCAGCCC

Statistical analyses. Statistical significance of differences between means was assessed by two-tailed $t$-test and $P<0.05$ was considered significant.

\section{References}

1. Monaco, A. P., Bertelson, C. J., Liechti-Gallati, S., Moser, H. \& Kunkel, L. M. An explanation for the phenotypic differences between patients bearing partial deletions of the DMD locus. Genomics 2, 90-95 (1988).

2. Ervasti, J. M. \& Sonnemann, K. J. Biology of the striated muscle dystrophin-glycoprotein complex. Int. Rev. Cytol. 265, 191-225 (2008).

3. Minetti, G. C. et al. Functional and morphological recovery of dystrophic muscles in mice treated with deacetylase inhibitors. Nat. Med. 12, 1147-1150 (2006).

4. Colussi, C. et al. HDAC2 blockade by nitric oxide and histone deacetylase inhibitors reveals a common target in Duchenne muscular dystrophy treatment. Proc. Natl Acad. Sci. USA 105, 19183-19187 (2008).

5. Cacchiarelli, D. et al. MicroRNAs involved in molecular circuitries relevant for the Duchenne muscular dystrophy pathogenesis are controlled by the dystrophin/nNOS pathway. Cell. Metab. 12, 341-351 (2010).

6. Bladen, C. L. et al. The TREAT-NMD DMD Global Database: analysis of more than 7,000 Duchenne muscular dystrophy mutations. Hum. Mutat. 36, 395-402 (2015).

7. Harper, S. Q. et al. Modular flexibility of dystrophin: implications for gene therapy of Duchenne muscular dystrophy. Nat. Med. 8, 253-261 (2002).

8. Anthony, K. et al. Dystrophin quantification and clinical correlations in Becker muscular dystrophy: implications for clinical trials. Brain 134, 3547-3559 (2011).

9. Sironi, M. et al. Trans-acting factors may cause dystrophin splicing misregulation in BMD skeletal muscles. FEBS Lett. 537, 30-34 (2003).

10. Miyagoe-Suzuki, Y. \& Takeda, S. Gene therapy for muscle disease. Exp. Cell Res. 316, 3087-3092 (2010).

11. Goyenvalle, A., Seto, J. T., Davies, K. E. \& Chamberlain, J. Therapeutic approaches to muscular dystrophy. Hum. Mol. Genet. 20, R69-R78 (2011).

12. Foster, H., Popplewell, L. \& Dickson, G. Genetic therapeutic approaches for Duchenne muscular dystrophy. Hum. Gene. Ther. 23, 676-687 (2012).

13. Douglas, A. G. L. \& Wood, M. J. A. Splicing therapy for neuromuscular disease. Mol. Cell. Neurosci. 56, 169-185 (2013).

14. Koo, T. \& Wood, M. J. Clinical trials using antisense oligonucleotides in duchenne muscular dystrophy. Hum. Gene. Ther. 24, 479-488 (2013).

15. Arechavala-Gomeza, V., Khoo, B. \& Aartsma-Rus, A. Splicing modulation therapy in the treatment of genetic diseases. Appl. Clin. Genet. 7, 245-252 (2014).

16. Delaporte, C., Dehaupas, M. \& Fardeau, M. Comparison between the growth pattern of cell cultures from normal and Duchenne dystrophy muscle. J. Neurol. Sci. 64, 149-160 (1984). 
17. Buckingham, M. \& Rigby, P. W. J. Gene regulatory networks and transcriptional mechanisms that control myogenesis. Dev. Cell 28, 225-238 (2014).

18. Twayana, S. et al. Biogenesis and function of non-coding RNAs in muscle differentiation and in Duchenne muscular dystrophy. Biochem. Soc. Trans. 41, 844-849 (2013).

19. Cacchiarelli, D. et al. miR-31 modulates dystrophin expression: new implications for Duchenne muscular dystrophy therapy. EMBO Rep. 12, 136-141 (2011).

20. Denti, M. A. et al. Long-term benefit of adeno-associated virus/antisensemediated exon skipping in Dystrophic mice. Hum. Gene Ther. 19, 601-608 (2008).

21. Cazzella, V. et al. Exon 45 skipping through U1-snRNA antisense molecules recovers the Dys-nNOS pathway and muscle differentiation in human DMD myoblasts. Mol. Ther. 20, 2134-2142 (2012).

22. Aartsma-Rus, A. et al. Targeted exon skipping as a potential gene correction therapy for Duchenne muscular dystrophy. Neuromuscul. Disord. 12, S71-S77 (2002).

23. Cartegni, L., Wang, J., Zhu, Z., Zhang, M. Q. \& Krainer, A. R. ESEfinder: a web resource to identify exonic splicing enhancers. Nucleic Acids Res. 31, 3568-3571 (2003).

24. Gromak, N., Matlin, A. J., Cooper, T. A. \& Smith, C. W. Antagonistic regulation of alpha-actinin alternative splicing by CELF proteins and polypyrimidine tract binding protein. RNA 9, 443-456 (2003).

25. Leroy, O. et al. ETR-3 represses Tau exons $2 / 3$ inclusion, a splicing event abnormally enhanced in myotonic dystrophy type I. J. Neurosci. Res. 84, 852-859 (2006).

26. Charlet, B. N., Logan, P., Singh, G. \& Cooper, T. A. Dynamic antagonism between ETR-3 and PTB regulates cell type-specific alternative splicing. Mol. Cell. 9, 649-658 (2002).

27. Anant, S. et al. Novel role for RNA- binding protein CUGBP2 in mammalian RNA editing. CUGBP2 modulates $\mathrm{C}$ to $\mathrm{U}$ editing of apolipoprotein $\mathrm{B}$ mRNA by interacting with apobec- 1 and ACF, the apobec-1 complementation factor. J. Biol. Chem. 276, 47338-47351 (2001).

28. Li, D., Bachinski, L. L. \& Roberts, R. Genomic organization and isoformspecific tissue expression of human NAPOR (CUGBP2) as a candidate gene for familial arhythmogenic right ventricular dysplasia. Genomics 74, 396-401 (2001).

29. Ladd, A. N., Charlet, N. \& Cooper, T. A. The CELF family of RNA binding proteins is implicated in cell-specific and developmentally regulated alternative splicing. Mol. Cell. Biol. 21, 1285-1296 (2001).

30. Shen, S. et al. MATS: a Bayesian framework for flexible detection of differential alternative splicing from RNA-Seq data. Nucleic Acids Res. 40, e61-e61 (2012).

31. Boussadia, O., Jacquemin-Sablon, H. \& Dautry, F. Exon skipping in the expression of the gene immediately upstream of N-ras (unr/NRU). Biochim. Biophys. Acta 1172, 64-72 (1993).

32. Bian, Y. et al. Tannic acid facilitates expression of the polypyrimidine tract binding protein and alleviates deleterious inclusion of CHRNA1 exon P3A due to an hnRNP H-disrupting mutation in congenital myasthenic syndrome. Hum. Mol. Genet. 18, 1229-1237 (2009).

33. Incitti, T. et al. Exon skipping and Duchenne muscular dystrophy therapy: selection of the most active U1 snRNA antisense able to induce dystrophin exon 51 skipping. Mol. Ther. 18, 1675-1682 (2010).

34. Savkur, R. S., Philips, A. V. \& Cooper, T. A. Aberrant regulation of insulin receptor alternative splicing is associated with insulin resistance in myotonic dystrophy. Nat. Genet. 29, 40-47 (2001).

35. van den Bergen, J. C. et al. Dystrophin levels and clinical severity in Becker muscular dystrophy patients. J. Neurol. Neurosurg. Psychiatry 85, 747-753 (2014).
36. van Putten, M. et al. The effects of low levels of dystrophin on mouse muscle function and pathology. PLoS ONE 7, e31937 (2012).

37. Godfrey, C. et al. How much dystrophin is enough: the physiological consequences of different levels of dystrophin in the mdx mouse. Hum. Mol. Genet. 24, 4225-4237 (2015).

38. Nigro, G., Comi, L. I., Politano, L. \& Bain, R. J. The incidence and evolution of cardiomyopathy in Duchenne muscular dystrophy. Int. J. Cardiol. 26, 271-277 (1990).

39. Martone, J., De Angelis, F. G. \& Bozzoni, I. U1 snRNA as an effective vector for stable expression of antisense molecules and for the inhibition of the splicing reaction. Methods Mol. Biol. 867, 239-257 (2012).

40. Choi, J. et al. MyoD converts primary dermal fibroblasts, chondroblasts, smooth muscle, and retinal pigmented epithelial cells into striated mononucleated myoblasts and multinucleated myotubes. Proc. Natl Acad. Sci. USA 87, 7988-7992 (1990).

\section{Acknowledgements}

We thank M. Marchioni for technical support, Dr A. D'Amico for BMD biopsies and the EuroBioBank and Telethon Network of Genetic Biobanks (GTB12001F and GTB12001H) for providing biological samples. A special thank is also addressed to the GS $\Delta 44$ family. This work was partially supported by grants from: ERC-2013 (AdG 340172-MUNCODD), Telethon (GGP11149), Parent Project Italia, AFM-Telethon (17835), Epigen-Epigenomics Flagship Project, AriSLA full grant 2014 'ARCI', HFSP (RGP0009/2014) and Istituto Pasteur-Fondazione Cenci Bolognetti to I.B.

\section{Author contributions}

J.M. and F.B. performed most of the experiments for the analysis of CELF2a expression and exon skipping analysis, J.M. also contributed to the manuscript writing; I.L. performed RNAseq and carried out data analysis; M.M. performed CLIP assay; O.S. took care of myoblast differentiations and fibroblast trans-differentiations; E.P. and L.P. followed the patient and provided muscle and skin biopsies; and I.B. coordinated the research and wrote the paper.

\section{Additional information}

Accession codes: RNA sequencing reads were deposited at Gene Expression omnibus (GSE70389).

Supplementary Information accompanies this paper at http://www.nature.com/ naturecommunications

Competing financial interests: The authors declare no competing financial interests.

Reprints and permission information is available online at http://npg.nature.com/ reprintsandpermissions/

How to cite this article: Martone, J. et al. The lack of the Celf2a splicing factor converts a Duchenne genotype into a Becker phenotype. Nat. Commun. 7:10488 doi: 10.1038/ncomms10488 (2016).

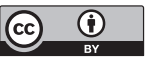

This work is licensed under a Creative Commons Attribution 4.0 International License. The images or other third party material in this article are included in the article's Creative Commons license, unless indicated otherwise in the credit line; if the material is not included under the Creative Commons license, users will need to obtain permission from the license holder to reproduce the material. To view a copy of this license, visit http://creativecommons.org/licenses/by/4.0/ 